\title{
A parametric study on the dynamics of two-span roll-to-roll microcontact printing system
}

\author{
SAJID $\mathrm{ALI}^{1, *}$ and MUHAMMAD A HAWWA ${ }^{2}$ \\ ${ }^{1}$ Mechanical and Energy Engineering Department, Imam Abdulrahman Bin Faisal University, Dammam 31441, \\ Saudi Arabia \\ ${ }^{2}$ King Fahd University of Petroleum and Minerals, Dhahran 31261, Saudi Arabia \\ e-mail: sakzada@iau.edu.sa; drmafh@kfupm.edu.sa
}

MS received 19 October 2018; revised 5 March 2019; accepted 12 March 2019; published online 25 April 2019

\begin{abstract}
The recent trend towards micro-patterning of thin film webs brought stricter manufacturing tolerances than traditionally admissible vibration levels. Therefore, one has to consider roll-to-roll (R2R) systems with their system dynamics model encompassing all systems' components, namely, the axially moving web(s), the rotating rolls and other accessories. In this study, the dynamic characteristics of an axially moving web in a two-span R2R system are investigated. Influence of geometrical parameters on the R2R dynamics and the parametric resonances of the moving web is presented. The time variations of web axial speed and webtransmitted tension are rigorously obtained by solving the web tension-roller angular speed equation, which is obtained from the conservation of mass law. The effects of system parameters such as length of the web, inertia of idler roll and radius of the rewinding/unwinding roll on the angular speed and web-transmitted tension have been discussed. The axially moving web was modelled as a string, which was mathematically represented by a second-order hyperbolic partial differential equation. The effect of the frequency of the disturbance at the roll on the web dynamics is also discussed. The transverse vibration response at selected points on the web shows higher frequency fluctuations corresponding to lower transport axial speed. It is noted that instability in the transverse response will occur when the first and second frequencies of the oscillation converge.
\end{abstract}

Keywords. Roll-to-roll system's dynamics; axially moving web; parametric instability; precise microcontact printing.

\section{Introduction}

Roll-to-roll (R2R) web processing is traditionally the method of choice for high-rate automated production of papers, textiles, sheet metals, thin polymers and laminated composites. $\mathrm{R} 2 \mathrm{R}$ production has a great advantage of guaranteeing a speedy fabrication with standardized quality. Limiting factors in R2R manufacturing include excessive web stretching and web wrinkling, which are normally avoided using tension control schemes. Another important factor that influences the quality of web processing is mechanical vibration, which can naturally arise as an unavoidable phenomenon of system's dynamics. Mechanical vibrations in R2R systems can be root caused at the rolls if they have out of round shape, are out of balance, are misaligned, accelerate, decelerate or stop. Web vibrations can also be caused by intermittent processing style that typically characterizes material deposition, ink printing and physical and chemical curing.

*For correspondence
The introduction of lithographic processes into R2R manufacturing schemes is an emerging technology for making flexible electronic devices in mass quantities. The recent trend towards micro-patterning of thin film webs brought stricter manufacturing tolerances than these traditionally admissible vibration levels. Therefore, one has to consider R2R systems with their system dynamics model encompassing all systems' components, namely, the axially moving web(s), the rotating rolls and other accessories such as dancers and brakes. R2R systems dynamic models indicate clearly a tension-speed coupling that one cannot ignore. Web tension regulation in R2R system was considered for the first time in [1] and subsequently in [2], where models of web tension are presented based on the methods from continuum mechanics. Dynamic models for the unwind/rewind rolls and free web span from first principles using Newton's laws and conservation of mass principle were derived in $[3,4]$. The R2R dynamics model is based on the laws of physics: (i) Hooke's law allows for web elasticity, (ii) Coulomb's law explains the contact between the web and the roll, including friction, (iii) the mass conservation 


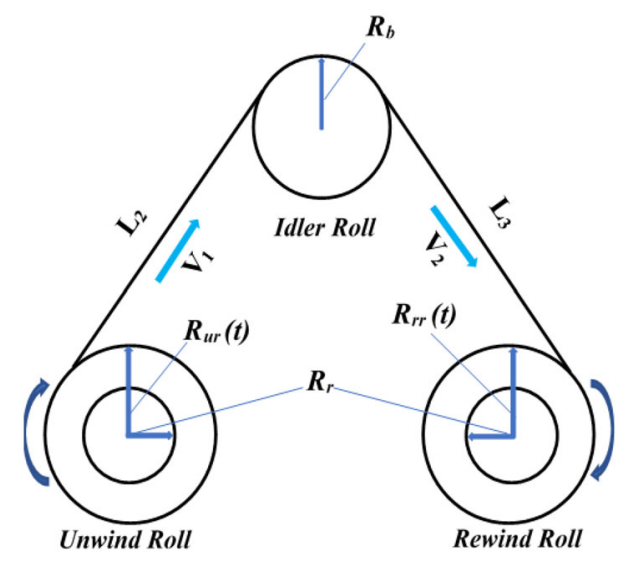

Figure 1. Two-span R2R system.

law allows for the coupling between the speed and the tension of the web and (iv) the second fundamental relation of dynamics accounts for variations of the rotating speeds [5]. Many researchers over the years applied this approach to develop the web tension-velocity model in an R2R system [6-14].

Browsing the literature on studying web vibrations, one can note that the problem has been treated in the context of axially moving material that is isolated from roll dynamics. This idealization has led to assuming "artificial" web tension and web speed profiles. In several cases, the idyllic conditions of constant tension and constant speed have been assumed [15-27]. Initials studies on the axially moving materials were of Sack [15] and Archibald and Emslie [17]. In [15, 17], the dynamic behaviour of axially moving string under constant speed and tension was investigated. A travelling chain was considered as an axially moving string and the natural frequencies were determined in [16]. Lateral vibration and stable operation conditions for an axially moving bandsaw blade were determined in [18]. Wickert and Mote [19] presented the transverse vibration of both axially moving string and beam using the Green's function method and was able to find the critical transport speed in both cases. It has been shown by Horssen [20] and Horssen and Ponomareva [23] that the initial-boundary value problem of an axially moving string can be solved using the method of Laplace transform. Influence of viscous damping on the natural frequencies and free vibration of an axially moving string was investigated in [21]. The method of multiple scales was applied to study the transverse response of parametrically excited axially moving visco-elastic belt under constant speed and tension in [22]. Stability analysis was performed for the no-linear vibration of moving string by Wang using the eigenvalue approach in [24] and multiplescale method in [26]. For a visco-elastic string, Zhang [25] discussed the periodicity of the transverse motion through Poincare maps. The Fourier-Galerkin-Newton
(FGN) method was used to show the dependences between axial speed and natural frequencies of the nonlinear free response of axially moving band by Koivurova [27]. Yusuf et al [28] studied the dynamic behaviour of multi-span Timoshenko beam with multiple spring-mass system. A finite-element model was developed by Reddy [29] to investigate the dynamic characteristics of a Timoshenko beam. To minimize the computational constraints by reducing the degrees of freedom, spectral element models (SEM) were applied on the transverse vibration of axially moving beams [30, 31]. To avoid the discretization of the governing hyperbolic partial differential equation (HPDE), the method of multiple scales was applied to investigate the dynamic stability and nonlinear free transverse vibration of axially moving viscoelastic and elastic beams [32-37].

Transverse vibration of an axially moving web can be excited by transverse displacements at the rolls, due to rolls eccentricity and other web imperfections. If the displacement at the roll is of harmonic nature, it may cause parametric resonances, which adversely affect the response of the system and may lead to failure of the system. In order to achieve an optimal design of the systems, the major task is to evaluate and assess all possible resonance frequencies and to keep them away from the natural frequencies of the system. Also, one has to consider R2R systems with their system dynamics model encompassing all systems' components, namely, the axially moving web(s), the rotating rolls and other accessories.

In this paper, it is intended to rigorously consider the coupling between the R2R dynamics and the transverse vibration of the web between the rolls. One does not need to assume simply supported boundary conditions for the vibration problem since the unwinding and rewinding rolls enter the analysis as part of the overall model. This comprehensive approach couples the physics-based variations of axial web tension with angular roll velocities to the web vibration-imposed fluctuations. The geometry of the R2R system has an influence on the end product and must be considered for understanding the complete dynamics of the system. Hence, the effects of system parameters such as the length of the web, moment of inertia of the idler roll and the radius of the rewinding/unwinding rolls on the angular speed and the web-transmitted tension are analysed. The effect of the frequency of the disturbance at the roll on the web dynamics in terms of amplitude-response is also presented.

\section{Mathematical model and solution}

In order to study the web transverse vibration in this $\mathrm{R} 2 \mathrm{R}$ system, one has to consider first the dynamic behaviour of this system that leads to establishing the relationship between the rotational motion of the rolls and the tension 
Table 1. Parameters of two-span system.

\begin{tabular}{lcc}
\hline Sl. no. & Parameter & Value \\
\hline 1 & Density of the web material & $8190 \mathrm{~kg} / \mathrm{m}^{3}$ \\
2 & Density of the roller material & $8050 \mathrm{~kg} / \mathrm{m}^{3}$ \\
3 & Thickness of the web & $0.000275 \mathrm{~m}$ \\
4 & Width of the web & $0.1 \mathrm{~m}$ \\
5 & Modulus of elasticity of web & $117 \mathrm{GPa}$ \\
6 & Radius of the unwinding and & $0.04 \mathrm{~m}$ \\
& rewinding rolls & \\
7 & Initial radius of unwinding & $0.15 \mathrm{~m}$ \\
8 & Initial radius of rewinding & $0.04 \mathrm{~m}$ \\
11 & Frictional torque at roll & $0.004 \mathrm{Nm}$ \\
14 & Moment of inertia of idler roll $\left(J_{b}\right)$ & $0.001 \mathrm{~kg} \mathrm{~m}$ \\
\hline
\end{tabular}

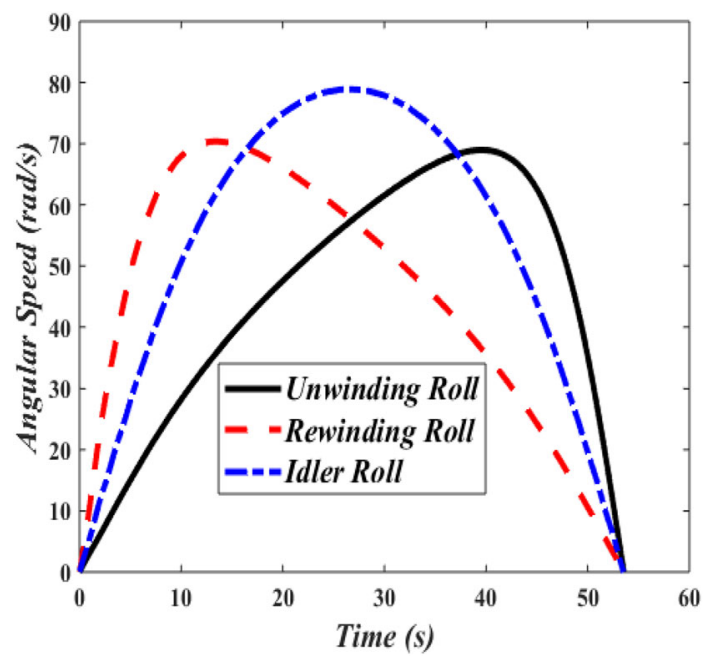

Figure 2. Angular velocity variation of rolls.

transmitted into the axially moving web. Having established the velocity-tension relationship, one can then consider the equation of transverse vibrational motion of the web.

\section{$2.1 R 2 R$ dynamic model}

A schematic of a two-span R2R system is shown in figure 1. Following the mathematical models of R2R systems presented by Brandenburg [38] and Koç et al [5], by assuming small width of the web in comparison with its length and considering small bending stiffness with no slip condition at the boundaries (rolls), the web tension-velocity relations for each span are given by

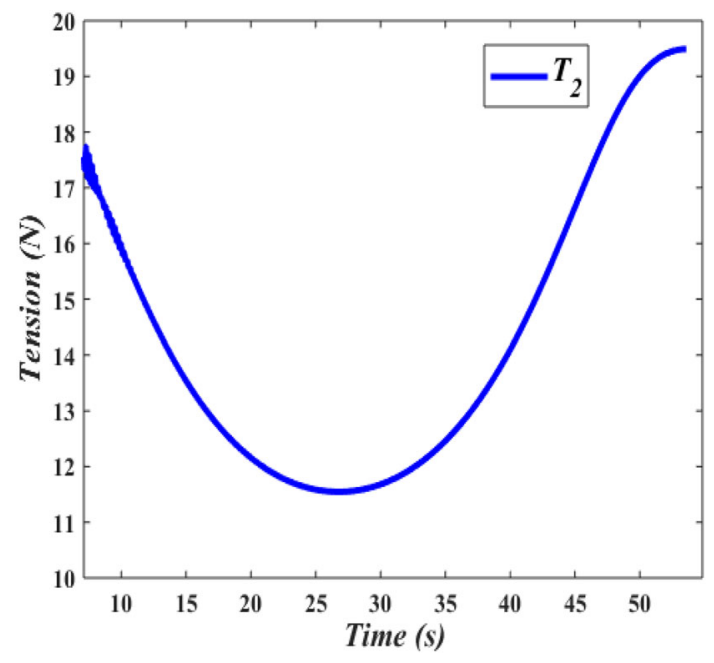

Figure 3. Web-transmitted tension $\left(T_{2}\right)$ variation with time.

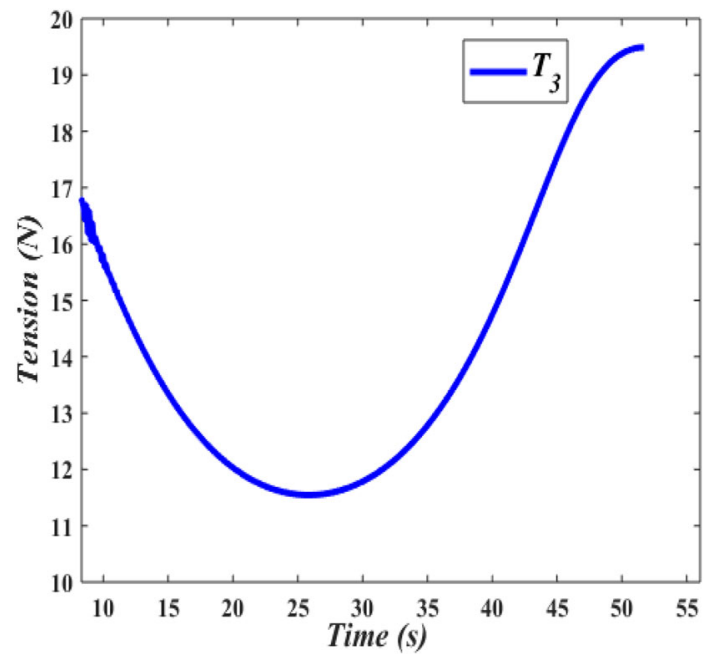

Figure 4. Web-transmitted tension $\left(T_{3}\right)$ variation with time.

$$
\begin{gathered}
\frac{\mathrm{d} T_{2}}{\mathrm{~d} t} \approx \\
{\left[\frac{R_{u r}}{L_{2}} T_{2}-\frac{E A R_{u r}}{L_{2}}\right] \Omega_{u r}} \\
+\left[\frac{R_{b}}{L_{2}} T_{3}-\frac{2 R_{b}}{L_{2}} T_{2}+\frac{E A R_{b}}{L_{2}}\right] \Omega_{b} \\
\frac{\mathrm{d} T_{3}}{\mathrm{~d} t} \approx\left[\frac{R_{b}}{L_{3}} T_{2}-\frac{2 R_{b}}{L_{3}} T_{3}-\frac{E A R_{b}}{L_{3}}\right] \Omega_{b}+\left[\frac{R_{r r}}{L_{3}} T_{3}+\frac{E A R_{r r}}{L_{3}}\right] \Omega_{r r}
\end{gathered}
$$

where " $E$ " is the modulus of elasticity of an elastic web, and " $A$ " is the cross-sectional area of the web. $T_{2}$ is the axial tension of unwind roll and idler roll, whereas $T_{3}$ is the axial tension of idler roll and rewind roll. Let us then focus on the forces acting on a roll; one has to consider (i) the inertial torque, (ii) the torque caused by the web tension, 


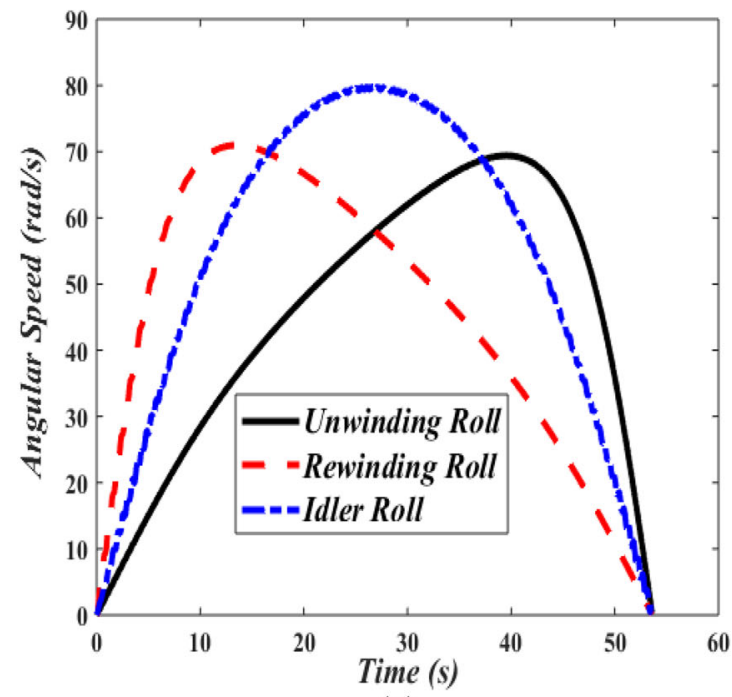

(a)

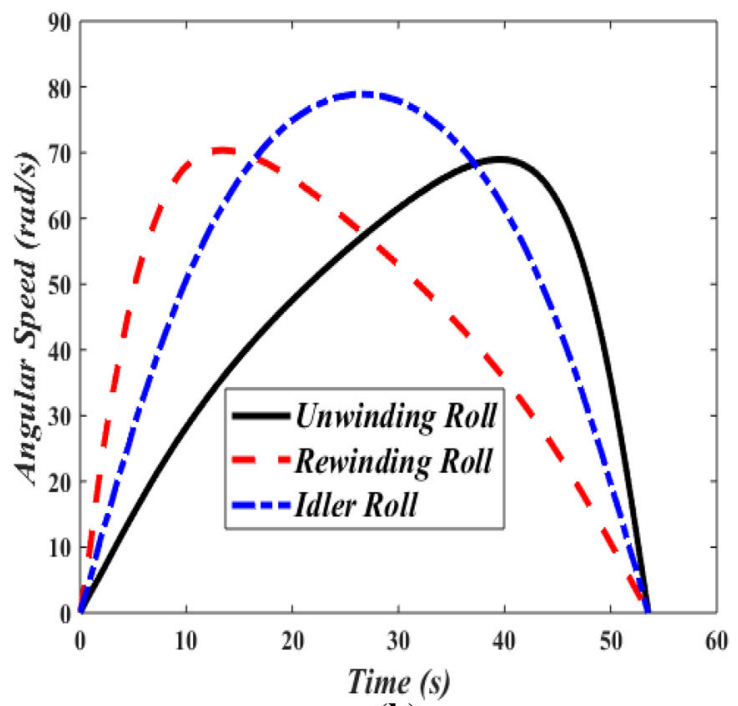

(b)

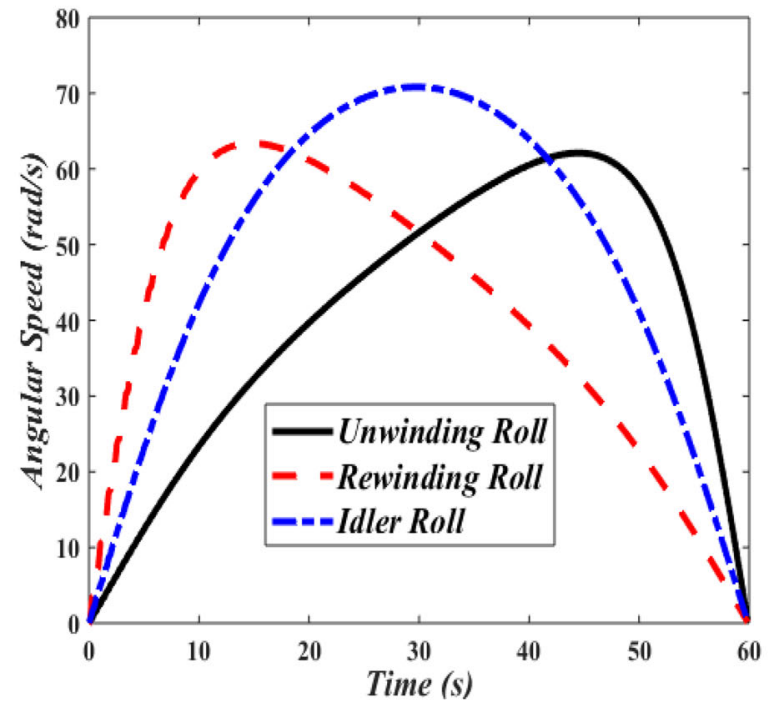

(c)

Figure 5. Angular velocity variation of rolls: (a) $J_{b}=0.0001$, (b) $J_{b}=0.001$ and (c) $J_{b}=0.01$.

(iii) the motor torque and (iv) the friction torque. Assuming slowly varying roll inertia and radii, the dynamic equilibrium equation of each roll can then be written as follows:

$$
\begin{gathered}
\frac{\mathrm{d}}{\mathrm{d} t}\left(J_{u r} \Omega_{u r}\right)=R_{u r} T_{2}+B_{1} \Omega_{u r}-C_{1} \\
\frac{\mathrm{d}}{\mathrm{d} t}\left(J_{b} \Omega_{b}\right)=R_{b}\left(T_{3}-T_{2}\right)+B_{2} \Omega_{b}-C_{2} \\
\frac{\mathrm{d}}{\mathrm{d} t}\left(J_{r r} \Omega_{r r}\right)=-R_{r r} T_{3}+B_{3} \Omega_{r r}-C_{3}
\end{gathered}
$$

where $\Omega_{u r}, \Omega_{b}, \Omega_{r r}$ are the angular speeds and $R_{u r}, R_{b}, R_{r r}$ are radii of unwinder, idler and rewinder roll, respectively. Inertia of idler roll, $J_{b}$, is constant whereas inertia variation of unwinder and rewinder roll is given by

$$
\begin{aligned}
& J_{u r}(t)=\frac{1}{2} \pi W\left[R_{r}^{4}\left(\rho_{r}-\rho_{w}\right)+\rho_{w}\left[R_{u r}(t)\right]^{4}\right] \\
& J_{r r}(t)=\frac{1}{2} \pi W\left[R_{r}^{4}\left(\rho_{r}-\rho_{w}\right)+\rho_{w}\left[R_{r r}(t)\right]^{4}\right]
\end{aligned}
$$

where $\rho_{r}$ is the density of roller, $R_{r}$ is the radius of roller and $W$ is the width of each roller's core.

\subsection{Web dynamic model}

For modelling the vibration of the elastic web as an axially moving string, Hamilton's principle can be used to derive the equation of motion for the travelling string as [19] 


$$
\frac{\partial^{2} w}{\partial t^{2}}+2 v \frac{\partial^{2} w}{\partial t \partial x}+\left(\frac{\rho A v^{2}-T}{\rho A}\right) \frac{\partial^{2} w}{\partial x^{2}}+v \cdot \frac{\partial w}{\partial x}=0
$$

where $w$ is the transverse displacement and $v$ is the axial speed of the web. Normalizing (8) by utilizing $L$ as a spatial constant, $\sqrt{\frac{\rho A L^{2}}{T}}$ as a temporal parameter, and $\sqrt{\frac{T A}{\rho}}$ as a reference velocity transforms the equation of motion into the following dimensionless one:

$$
\frac{\partial^{2} w_{*}}{\partial t_{*}^{2}}+2 v_{*} \frac{\partial^{2} w_{*}}{\partial t_{*} \partial x_{*}}+\left(v_{*}^{2}-1\right) \frac{\partial^{2} w_{*}}{\partial x_{*}^{2}}+v_{*} \frac{\partial w_{*}}{\partial x_{*}}=0 .
$$

Note that variables with stars are dimensionless, and $v_{*}$ is a dimensionless transport speed that is the ratio of physical velocity to wave velocity. Equation (9) is a HPDE for the transverse vibration of the axially moving string. The disturbances at the boundary are given in the following equation:

$$
\left.\begin{array}{l}
w_{*}(0, t)=0.01 \sin (\Omega t) \\
w_{*}(0, t)=0
\end{array}\right\} .
$$

For these boundary conditions, finite-difference approach is used to transform the second-order HPDE into second-order ordinary differential equations (ODEs). Applying central difference scheme to discretize equation of motion results in $n$ second-order ODEs (11), where $n$ is the total number of spatial points and $\mathrm{d} x_{*}$ is the step size:

$$
\begin{aligned}
& \frac{\mathrm{d}^{2} w_{* 0}}{\mathrm{~d} t_{*}^{2}}=0.01 \Omega \cos (\Omega t) \\
& \frac{\mathrm{d}^{2} w_{* 1}}{\mathrm{~d} t_{*}^{2}}+\frac{v_{*}}{\mathrm{~d} x_{*}}\left(\frac{\mathrm{d} w_{* 2}}{\mathrm{~d} t_{*}}-\frac{\mathrm{d} w_{* 0}}{\mathrm{~d} t_{*}}\right) \\
& +\frac{\left(v_{*}^{2}-1\right)}{\mathrm{d} x_{*}^{2}}\left(w_{* 0}-2 w_{* 1}+w_{* 2}\right)+\frac{v_{*}}{2 \mathrm{~d} x_{*}}\left(w_{* 2}-w_{* 0}\right) \\
& =0, \frac{\mathrm{d}^{2} w_{* 2}}{\mathrm{~d} t_{*}^{2}}+\frac{v_{*}}{\mathrm{~d} x_{*}}\left(-\frac{\mathrm{d} w_{* 1}}{\mathrm{~d} t_{*}}+\frac{\mathrm{d} w_{* 3}}{\mathrm{~d} t_{*}}\right) \\
& +\frac{\left(v_{*}^{2}-1\right)}{\mathrm{d} x_{*}^{2}}\left(w_{* 1}-2 w_{* 2}+w_{* 3}\right)+\frac{v_{*}}{2 \mathrm{~d} x_{*}}\left(-w_{* 1}+w_{* 3}\right) \\
& =0 \text {, } \\
& \begin{array}{c}
\vdots \\
\vdots \\
\vdots \\
\frac{\mathrm{d}^{2} w_{*(n-1)}}{\mathrm{d} t_{*}^{2}}+\frac{v_{*}}{\mathrm{~d} x_{*}} \frac{\mathrm{d} w_{*(n-2)}}{\mathrm{d} t_{*}}+\frac{\left(v_{*}^{2}-1\right)}{\mathrm{d} x_{*}^{2}}\left(w_{*(n-2)}-2 w_{*(n-1)}\right) \\
+\frac{v_{*}^{*}}{2 \mathrm{~d} x_{*}} w_{*(n-2)}=0 .
\end{array}
\end{aligned}
$$

The system of equations (11) is numerically integrated to find the time-domain response of an axially moving web.

\section{Numerical solution and discussion}

A numerical simulation for describing the transfer of a certain amount of web material from the unwinding roll to the rewinding roll in a two-span R2R system is performed. Equations (1)-(5) are numerically integrated to find the time-domain description of R2R system's motion. Parameters of the system used in the simulation are given in table 1 .

The angular speeds of unwinding, idler and rewinding rolls vary with time as shown in figure 2 .

The angular velocity of the rewinding roll increases at a faster rate than that of the unwinding roll. This is because the rewinding roll initially has a lower moment of inertia than the unwinding roll. The opposite effect occurs after the two rolls reach the same angular velocity. The radii variations will be such that the unwinding roll radius shrinks to the value of the initial radius of the rewinding roll and the rewinding roll radius grows to the value of the initial radius of the unwinding roll within the same time. Axial tension in the web between two rolls in the first and the second span are shown in figures 3 and 4 .

The variation of the web-transmitted tension with time in both spans, shown in figures 3 and 4 , is initially transient, and then becomes smooth and slightly nonlinear.

\section{$3.1 R 2 R$ system's parameters}

The geometry of the R2R system has an influence on the end product. In this section, effects of system parameters such as the length of the web, moment of inertia of the idler roll and the radius of the rewinding/unwinding rolls on the angular speed and the web-transmitted tension are analysed. The effect of idler roll moment of inertia $\left(J_{b}\right)$ on angular speed of each roll is presented in figure 5 . Three different $J_{b}$ values, i.e. $J_{b}=0.0001,0.001$ and 0.01 , are considered.

Comparing the angular speeds in figure 5 indicates that increasing the idler roll moment of inertia leads to a reduced amplitude of the roll angular speed at the expense of the increased time required to transfer the web material from the unwinding roll to the rewinding roll.

Axial web tension in the first and second web spans at different idler roll moments of inertia is shown in figures 6 and 7.

Comparison of the variation in web-transmitted tension with time in both spans indicates that the magnitude of the tension is affected by the idler roll moment of inertia, and time span increases as we increase the idler roll moment of inertia. The effect of web length on the web-transmitted axial tension was studied. Comparison of web tension in both spans at the different web lengths $L=$ $1.9,1.5,1.2,0.95$ and $0.475 \mathrm{~m}$ is shown in figures 8 and 9 .

Comparative study shows that changing the web length does not affect the web-transmitted tension in both spans. 


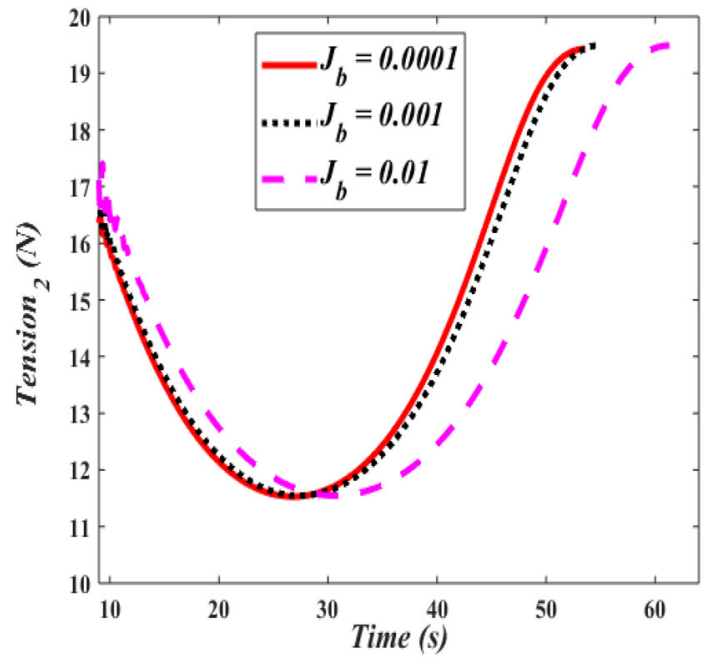

Figure 6. Comparing the web-transmitted tension $\left(T_{2}\right)$ at different idler roll inertias.

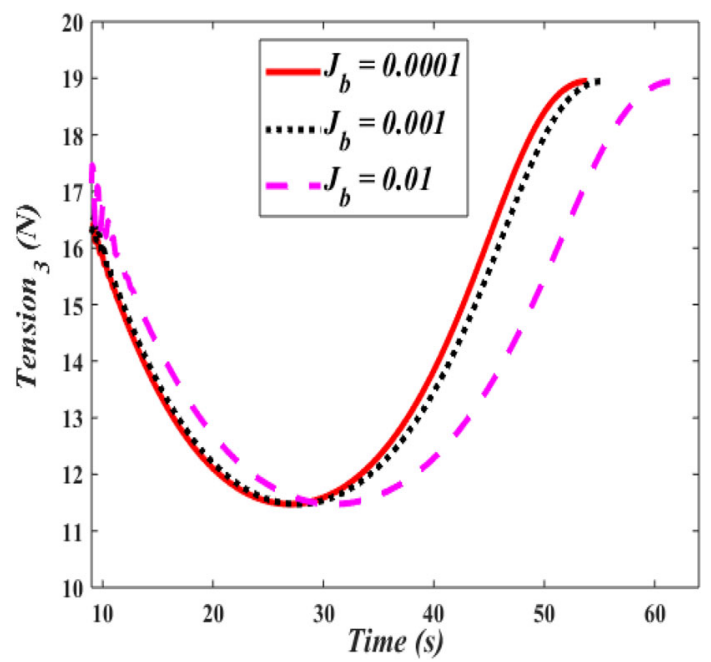

Figure 7. Comparing the web-transmitted tension $\left(T_{3}\right)$ at different idler roll inertias.

The effect of the rewinding roll radius on the angular speed and the web-transmitted tension was also studied. The angular speed of each roll is presented in figure 10 .

By reducing the rewinder roll radius, the angular velocity of the rewinding roll increases at a faster rate than that of the unwinding roll. This is because the rewinding roll initially has a lower moment of inertia than the unwinding roll. Furthermore, comparison of angular speeds in figure 10 indicates that reducing the rewinding roll radius leads to an increased amplitude in the roll angular speed and reduces the time required to transfer the web material from the unwinding roll to the rewinding roll. Axial tension in the web between the two rolls in the first and second span is shown in figures 11 and 12.

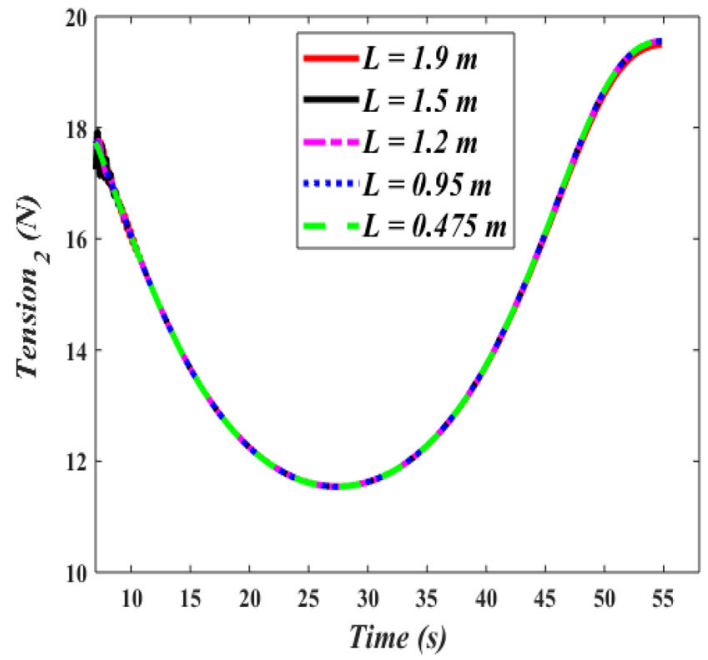

Figure 8. Web-transmitted tension $T_{2}$.

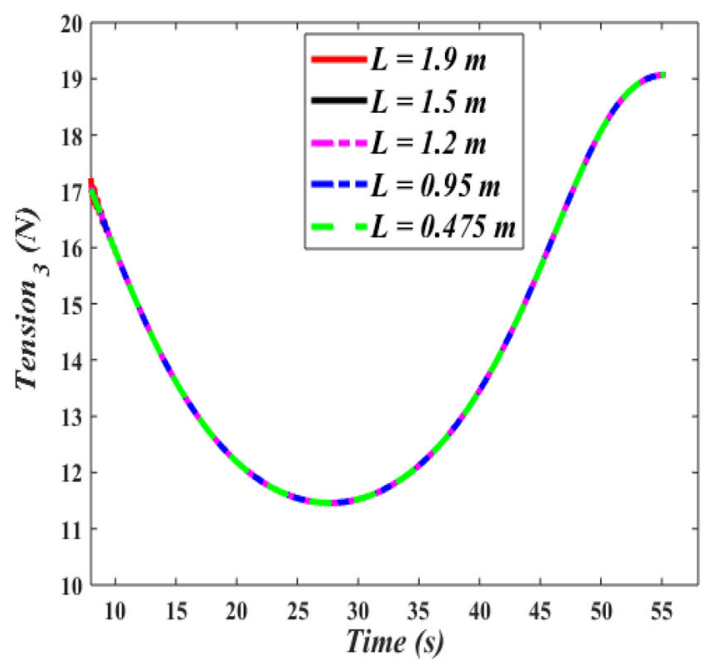

Figure 9. Web-transmitted tension $T_{3}$.

Comparison shows that reducing the rewinder radius not only reduces the time span but also increases the magnitude of the variation in axial tension. A closer look at the axial tension reveals that the magnitude of the web tension is increased by reducing the rewinding roll radius.

\subsection{Parametric analysis}

In this section, the effects of system's parameters on its response are investigated. Figures 13-17 depict the dynamic response of the system when excited with external disturbance of frequency $\Omega$. Response of the system is 


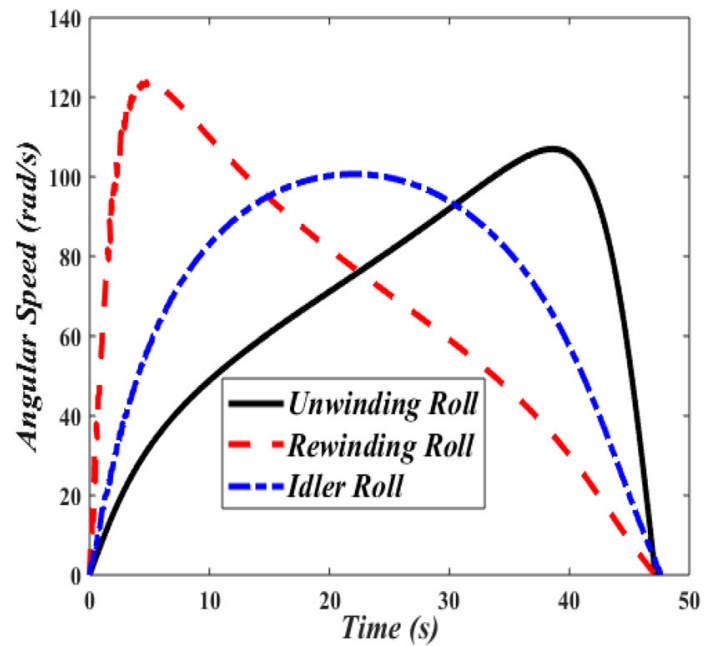

Figure 10. Time history of angular speed $\left(R_{u r}=0.04 \mathrm{~m}\right.$ and $R_{r r}$ $=0.02 \mathrm{~m}$ ).

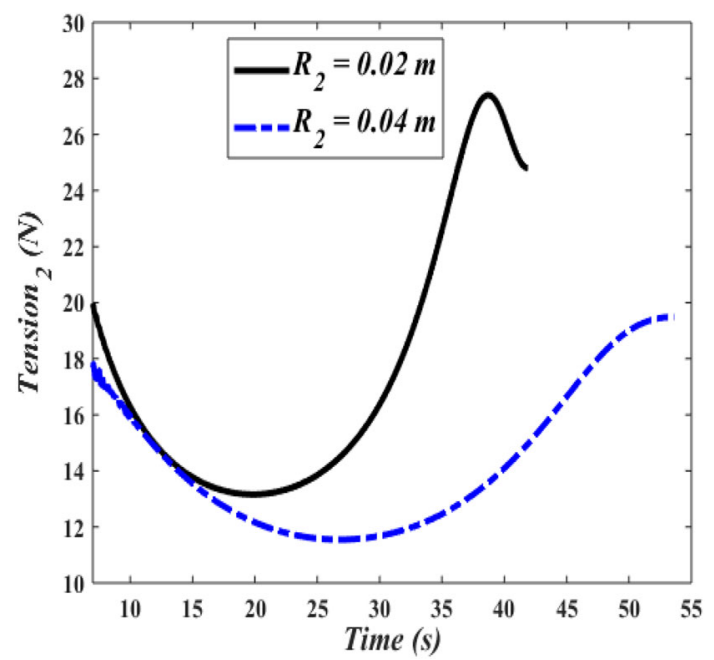

Figure 11. Web-transmitted tension $T_{2}\left(R_{r r}=0.02\right.$ and $\left.0.04 \mathrm{~m}\right)$.

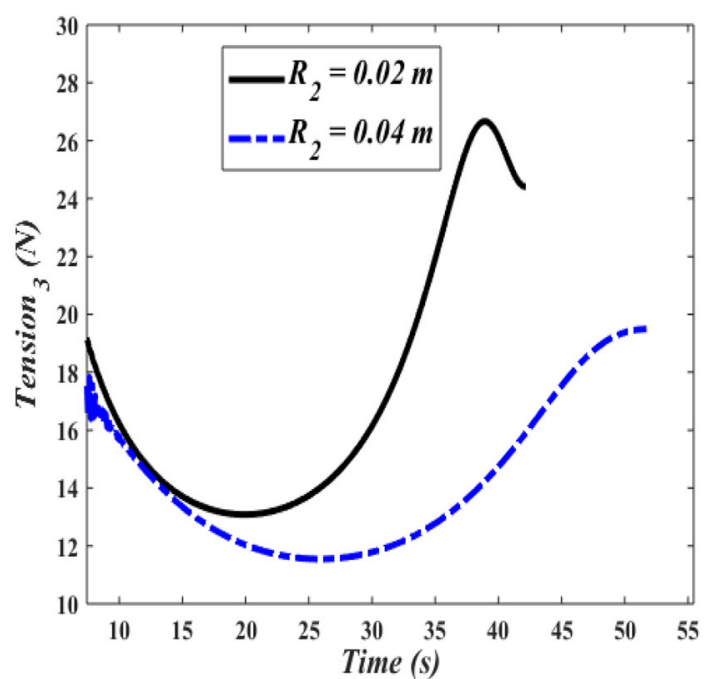

Figure 12. Web-transmitted tension $T_{3}\left(R_{r r}=0.02\right.$ and $\left.0.04 \mathrm{~m}\right)$.

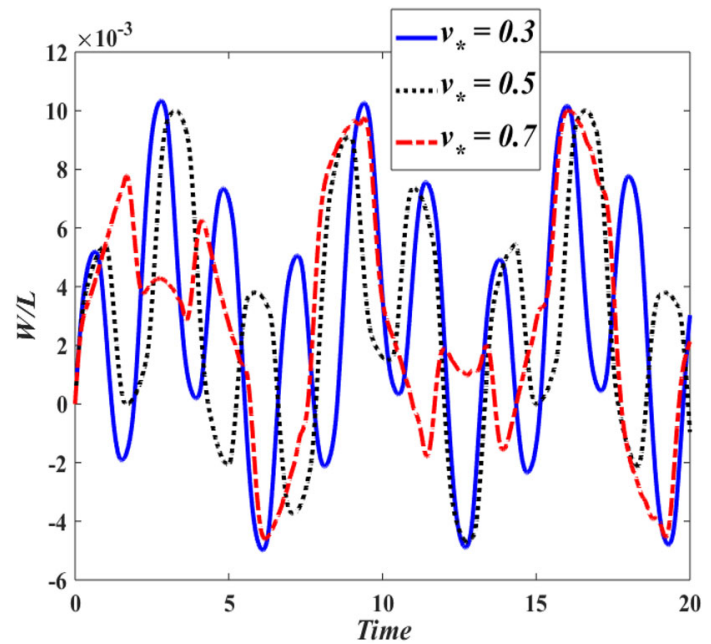

Figure 13. Transverse displacement at $x_{*}=0.5$ and $\Omega=0.6$ for webs having different speeds.

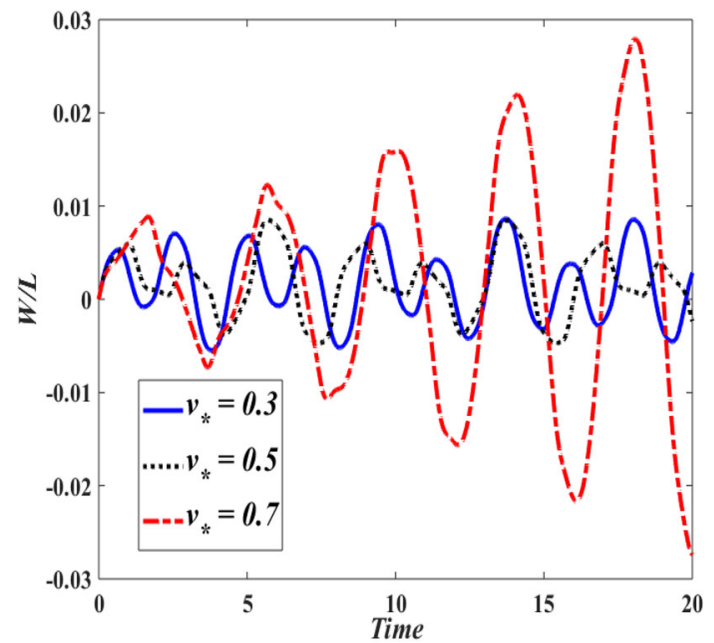

Figure 14. Transverse displacement at $x_{*}=0.5$ and $\Omega=1.2$ for webs having different speeds.

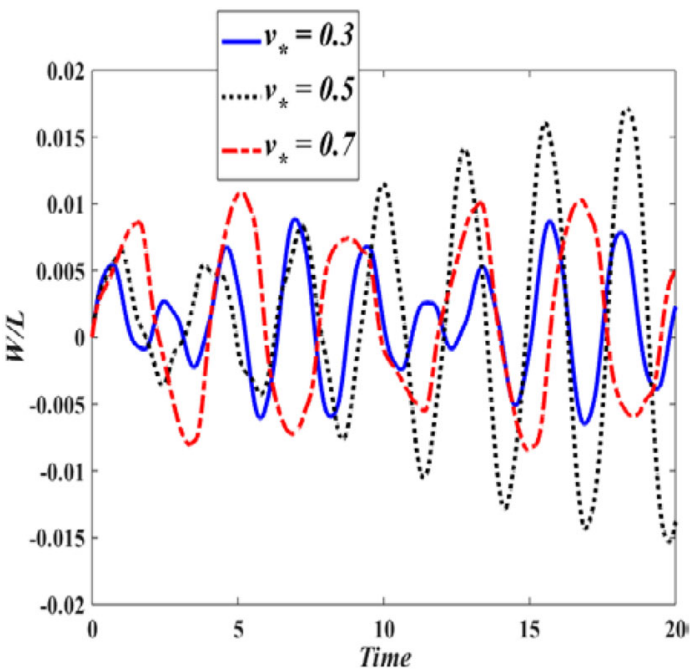

Figure 15. Transverse displacement at $x_{*}=0.5$ and $\Omega=1.4$ for webs having different speeds. 
monitored at mid web span $\left(x_{*}=0.50\right)$ and three different web speeds have been considered, i.e., $v_{*}=0.3,0.5$ and 0.7 .

Figure 13 depicts the dynamic response of the web at a lower excitation frequency. At a lower frequency, the response is stable for the given web axial speed. As we increase the excitation frequency, the response moves towards resonance. When excitation frequency is 1.2 the response of the system for axial speed of 0.7 becomes unstable while system stability is retained for other two speeds, shown in figure 14. As excitation frequency is increased to 1.4 and 1.7 the response becomes unstable at web speeds of 0.5 and 0.3 , respectively, while the other two responses remain stable for each case, shown in figures 15 and 16. The frequency-domain responses, obtained by

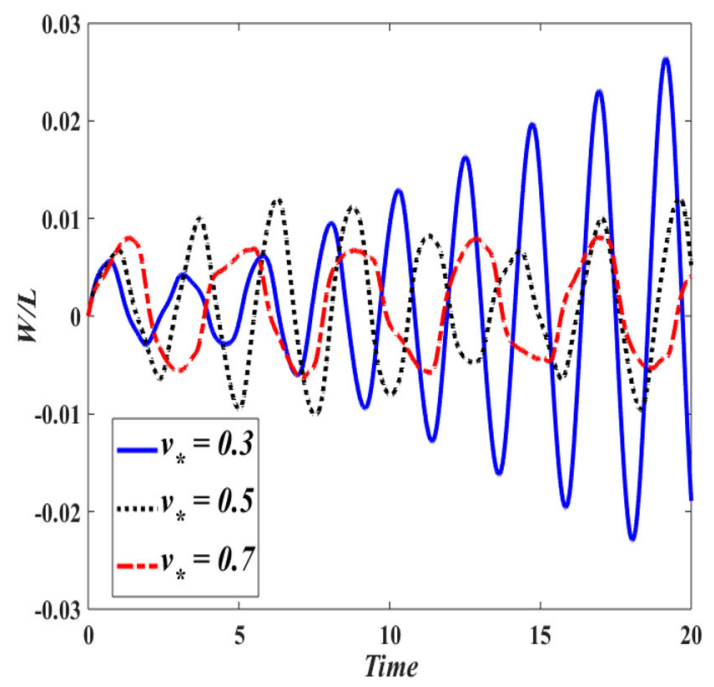

Figure 16. Transverse displacement at $x_{*}=0.5$ and $\Omega=1.7$ for webs having different speeds.

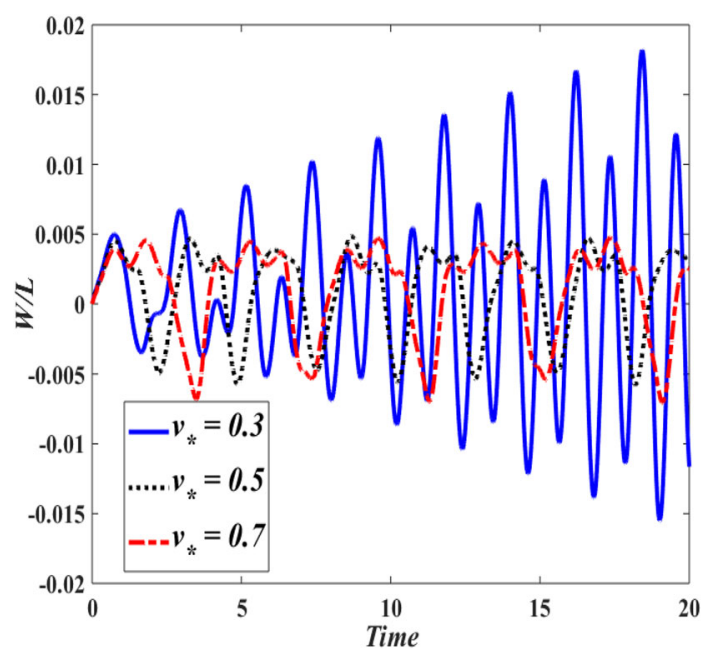

Figure 17. Transverse displacement at $x_{*}=0.5$ and $\Omega=3.4$ for webs having different speeds. applying Fast Fourier Transform, are shown in figures 1822.

The first and second frequencies of transverse vibration of the system at different axial speeds of the web and the external excitation frequency are shown. Response frequency is the highest at lowest speed and as web speed is increased, frequency response is shifted to lower values. It is noted that instability in the transverse response will occur when the first and second frequencies of the oscillation converge to same value, shown in figures 19-22.

3.2.1 The coupled web vibration In a multi-span $\mathrm{R} 2 \mathrm{R}$ system, boundary conditions are different for each span. In the unwinding span, the radius of the idler roll will remain constant, whereas the radius of the unwinding roll will

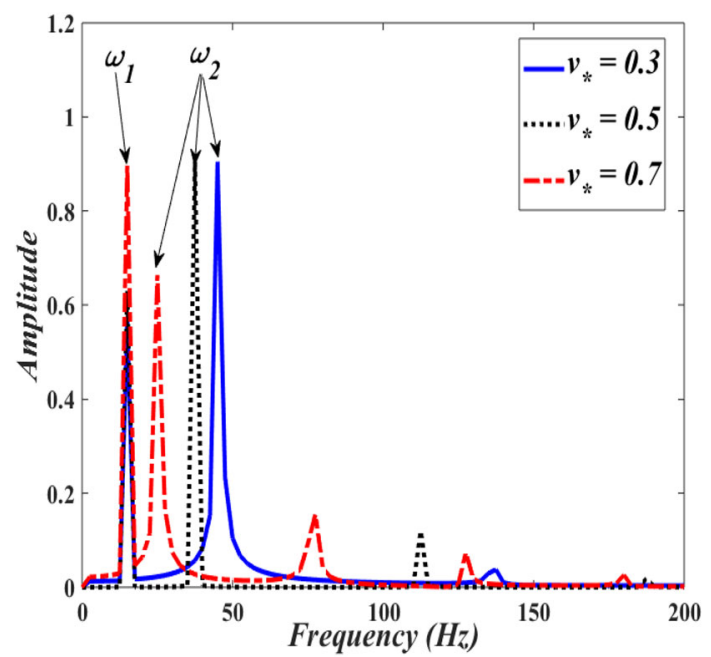

Figure 18. Frequency-domain response of displacement at $x_{*}=$ 0.5 and $\Omega=0.6$ for webs having different speeds.

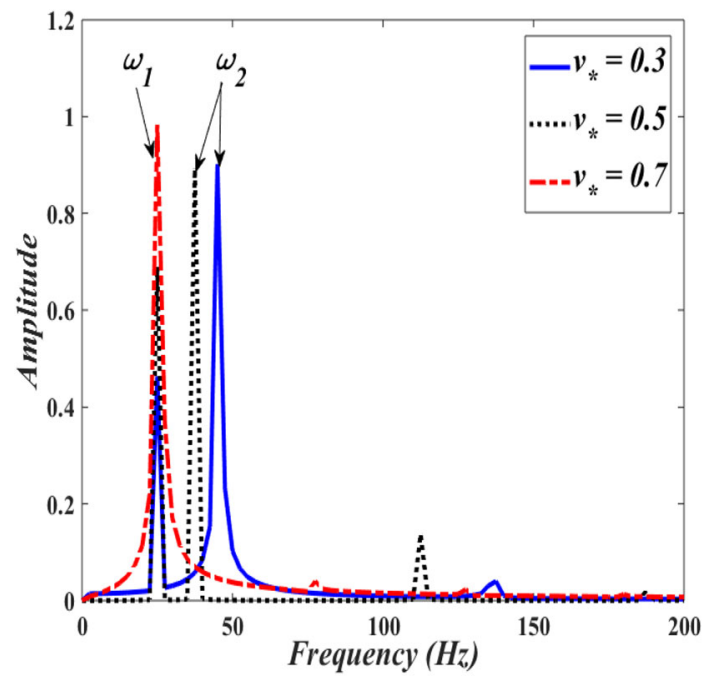

Figure 19. Frequency-domain response of displacement at $x_{*}=$ 0.5 and $\Omega=1.2$ for webs having different speeds. 
decrease (starting from the outer radius) with time, i.e. at the left boundary there is a varying transverse displacement. In contrast with the unwinding span, in the rewinding span the radius of the idler roll will remain constant, whereas the radius of the rewinding roll will increase with time, i.e. at the right boundary there is a varying transverse displacement.

Equations (11) describing the transverse vibration in axially moving web are solved together as a coupled system of five equations. Figure 23 shows the midspan displacement for the unwinding and rewinding span.

The responses presented in figure 23 indicate non-periodic oscillations. It is worth noting that the magnitude of the response constantly decreases as the displacement at the left boundary decreases (figure 23(a)).

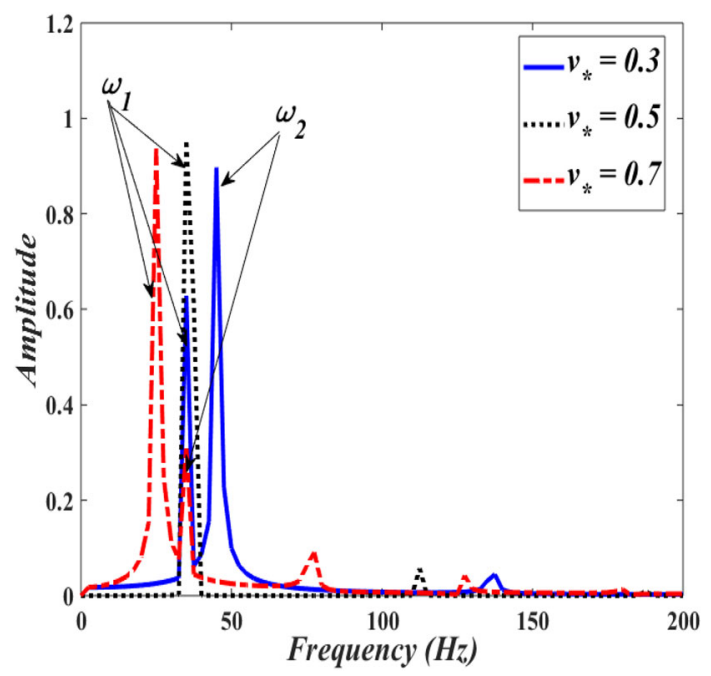

Figure 20. Frequency-domain response of displacement at $x_{*}=$ 0.5 and $\Omega=1.4$ for webs having different speeds.

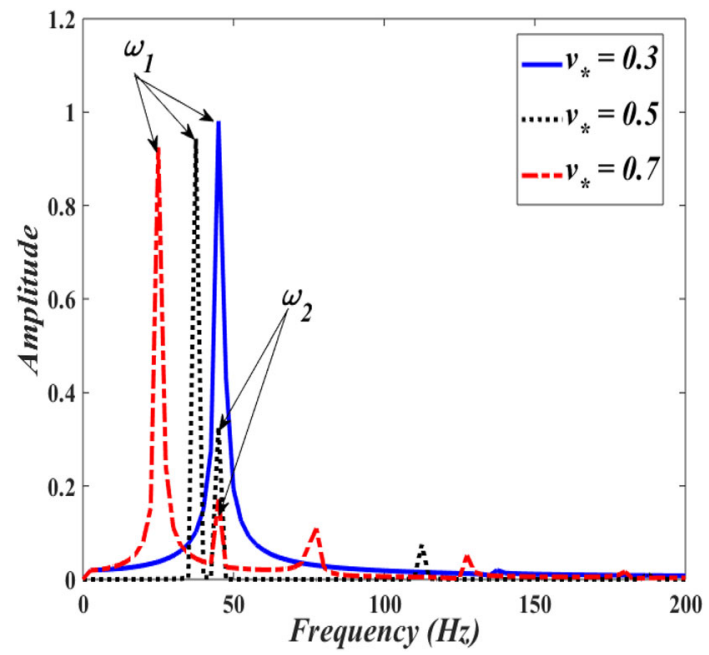

Figure 21. Frequency of response of displacement at $x_{*}=0.5$ and $\Omega=1.6$ for webs having different speeds.

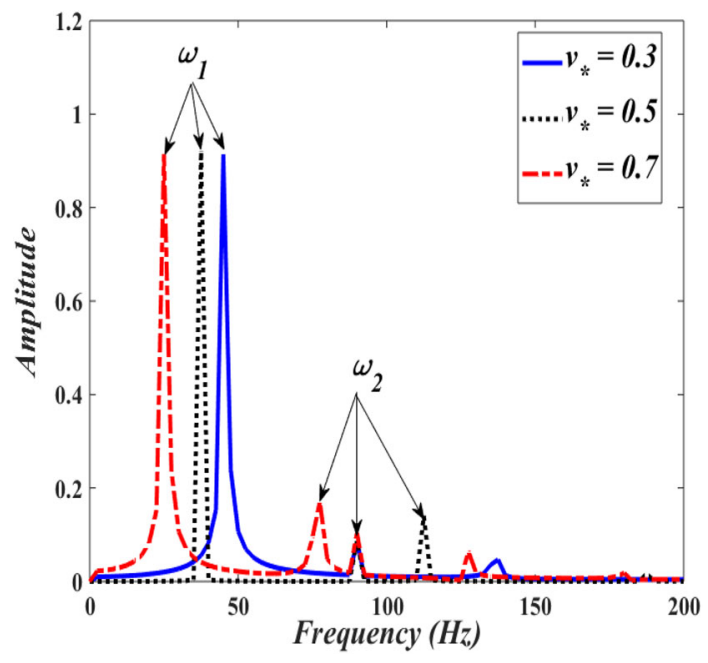

Figure 22. Frequency-domain response of displaceme at $x_{*}=$ 0.5 and $\Omega=3.4$ for webs having different speeds.

At the early and late phases of material transfer from the unwinding roll to the rewinding roll, the axial web speed changes rapidly with time, leading to high levels of frequency fluctuation in the dynamic response. The magnitude of the response constantly increases as the displacement at the right boundary increases (figure 23(b)). A low frequency response is noted in the middle of the web material transfer time interval, due to the slow change in the axial web speed with time. A slight difference can be observed in the web-transmitted tension variation with time upon comparing the R2R system's dynamic results (non-coupled, $\mathrm{NC}$ ) with those of the coupled system (C), as shown in figure 24.

\section{Conclusion}

The dynamic characteristics of an axially moving web in a two-span R2R system were investigated. Parametric study on the two web spans of three rolls was conducted to see the effects of web lengths, the moment of inertia of idler impression roll and the radii of the rewinding/unwinding rolls on the angular speed and web-transmitted tension. The axially moving web was modelled as a string, which was mathematically represented by a second-order HPDE. The $\mathrm{R} 2 \mathrm{R}$ dynamics was described by nonlinear coupled firstorder ODEs. It was found that the web-transmitted tension has undergone a short transient phase followed by a smooth nonlinear variation in time. While transferring a certain amount of web material from the unwinding roll to the rewinding roll, the angular speed of both rolls varied nonlinearly, attaining the same maximum angular speed within the time of web material transfer. Increasing the idler impression roll moment of inertia led to a reduced amplitude in the roll angular speed. Decreasing the length of one of the web spans did not affect tension profile, while it 


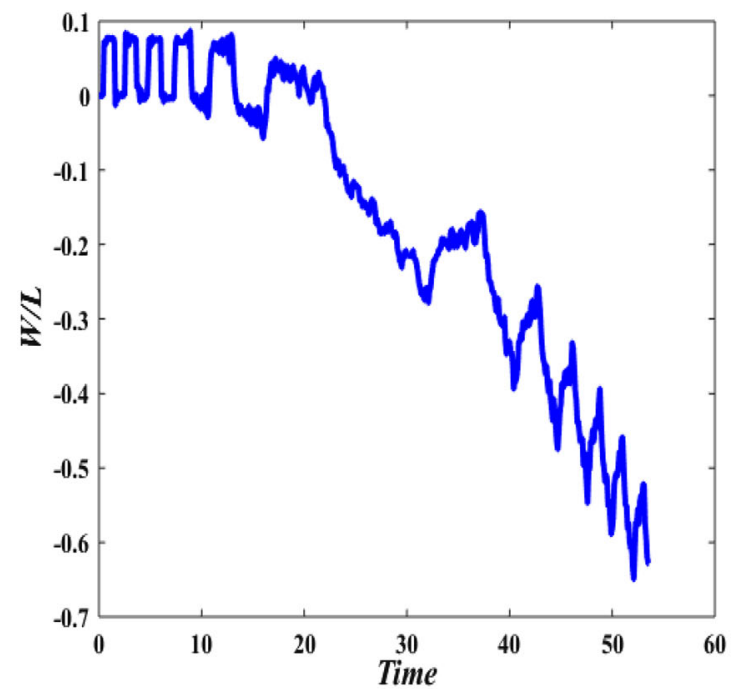

(a)

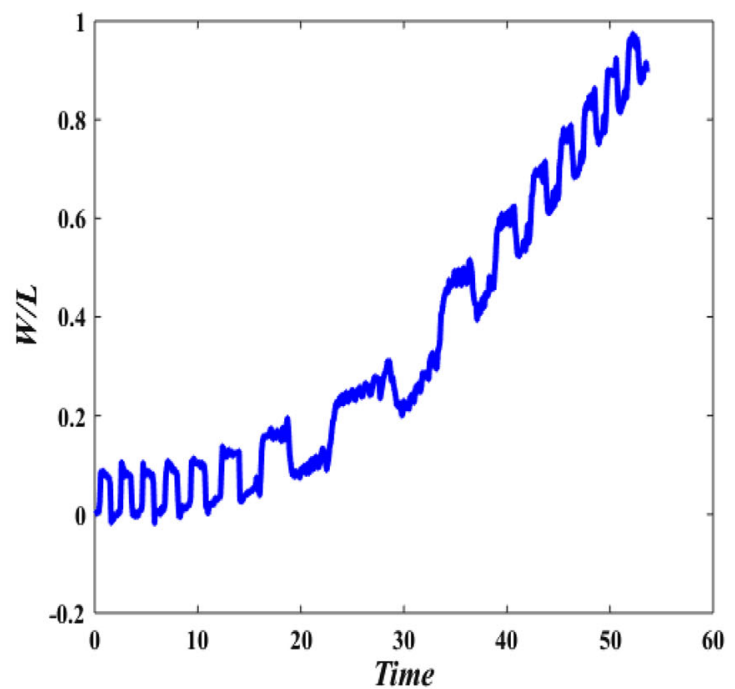

(b)

Figure 23. Transverse displacement at $x_{*}=0.5$ : (a) unwinding span and (b) rewinding span.

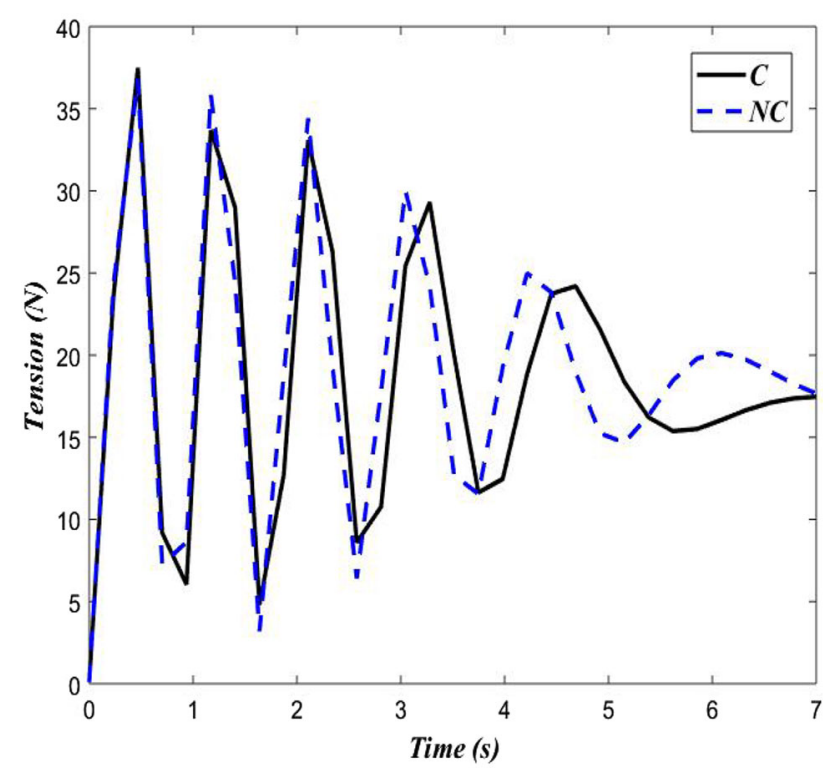

Figure 24. Comparison of web-transmitted tension (transient $T_{2}$ ).

slightly decreased the duration of the transient state. Reducing the rewinding roll radius to half of the unwinding radius increased the angular velocity of the rewinding roll at a faster rate and increased the magnitude of the fluctuations in the transient state. The effect of the external disturbance frequency on the dynamic response was investigated. It was observed that instability in transverse response occurred when the first and second frequencies of oscillation converged or when the second frequency of oscillation became a multiple of the first frequency of oscillation.

\section{Acknowledgement}

The authors would like to acknowledge the support provided by King Fahd University of Petroleum and Minerals (KFUPM) and Center for Clean Water and Clean Energy at MIT and KFUPM.

\section{References}

[1] Grenfell K 1964 Tension control on paper-making and converting machinery. IEEE Transactions on Applications and Industry 83: 234-240

[2] Brandenburg G 1972 The dynamics of elastic webs threading a system of rollers. Newspaper Techniques 12-25

[3] Whitworth D 1979 Tension variations in pliable material in production machinery. $\mathrm{PhD}$ Dissertation, Loughborough University of Technology

[4] Whitworth D and Harrison M 1983 Tension variations in pliable material in production machinery. Journal of Applied Mathematical Modeling 7: 189-196

[5] Koç H, Knittel D, Mathelin M and Abba G 2002 Modeling and robust control of winding systems for elastic webs. IEEE Transactions on Control Systems Technology 10(2): 197-208

[6] Koç H, Knittel D, Mathelin M and Abba G 1999 Modeling and control of an industrial accumulator in a web transport system. In: Proceedings of the European Control Conference (ECC)

[7] Koç H, Knittel D, Mathelin M and Abba G 1999 Web tension control in an industrial accumulator. In: Proceedings of the International Conference on Web Handling (IWEB5)

[8] Yun S, Han C and Chung J 2001 A study on the robust control algorithm for an axially moving film. KSME International Journal 15: 1207-1216

[9] Valenzuela A, Bentley J and Lorenz R 2003 Sensorless tension control in paper machines. IEEE Transactions on Industry Applications 39(2): 294-304 
[10] Chen C, Chang K and Chang C 2004 Modeling and control of a web-fed machine. Applied Mathematical Modelling 28: 863-876

[11] Laroche E and Knittel D 2005 An improved linear fractional model for robustness analysis of a winding system. Control Engineering Practice 13: 659-666

[12] Pagilla P, Siraskar N and Dwivedula R 2007 Decentralized control of web processing lines. IEEE Transactions on Control Systems Technology 15: 106-117

[13] Dou X and Wang W 2010 Robust control of multistage printing systems. Control Engineering Practice 18: 219-229

[14] Tran T and Choi K 2014 A back stepping-based control algorithm for multi-span roll-to-roll web system. The International Journal of Advanced Manufacturing Technology 70: 45-61

[15] Sack R A 1954 Transverse oscillations in traveling strings. British Journal of Applied Physics 5: 224-226

[16] Mahalingam S 1957 Transverse vibrations of power transmission chains. British Journal of Applied Physics 8: 145-148

[17] Archibald F R and Emslie A G 1958 The vibrations of a string having a uniform motion along its length. ASME Journal of Applied Mechanics 25: 347-358

[18] Naguleswaran S and Williams C J 1968 Lateral vibration of band-saw blades, pulley belts and the like. International Journal of Mechanical Sciences 10: 239-250

[19] Wickert J A and Mote C D 1990 Classical vibration analysis of axially moving continua. ASME Journal of Applied Mechanics 57: 738-744

[20] Horssen W T 2003 On the influence of lateral vibrations of supports for an axially moving string. Journal of Sound and Vibration 268: 323-330

[21] Jaksic N and Boltezar M 2005 Viscously damped transverse vibrations of an axially-moving string. Journal of Mechanical Engineering 51: 560-569

[22] Mockersturm E M and Guo J 2005 Nonlinear vibration of parametrically excited, viscoelastic, axially moving strings. Journal of Applied Mechanics 72: 374-380

[23] Horssen W T and Ponomareva S V 2005 On the construction of the solution of an equation describing an axially moving string. Journal of Sound and Vibration 287: 359-366

[24] Wang Y and Liu X 2005 Eigenvalue and stability analysis for transverse vibrations of axially moving strings based on Hamiltonian dynamics. Acta Mechanica Sinica 21: 485-494

[25] Zhang N H 2008 Dynamic analysis of an axially moving viscoelastic string by the Galerkin method using translating string eigenfunctions. Chaos, Solitons and Fractals 35: 291-302

[26] Wang Y, Liu X and Huang L 2008 Stability analyses for axially moving strings in nonlinear free and aerodynamically excited vibrations. Chaos, Solitons and Fractals 38: 421-429

[27] Koivurova H 2009 The numerical study of the nonlinear dynamics of a light, axially moving string. Journal of Sound and Vibration 320: 373-385

[28] Yusuf Y, Oktay D and Seval C 2008 Free vibrations of a multi-span Timoshenko beam carrying multiple spring-mass systems. Sadhana 34: 385-401

[29] Reddy J 1999 On the dynamic behaviour of the Timoshenko beam finite elements. Sadhana 24: 175-198

[30] Mokhtari A, Mirdamadi HR, Ghayour M and Sarvestan S 2016 Time/wave domain analysis for axially moving pre-stressed nanobeam by wavelet-based spectral element method. International Journal of Mechanical Sciences 105: 58-69

[31] Kim T and Lee U 2017 Dynamic analysis of a multi-span beam subjected to a moving force using the frequency domain spectral element method. Computers and Structures 192: 181-195

[32] Sahoo B, Panda L N and Pohit G 2015 Two-frequency parametric excitation and internal resonance of a moving viscoelastic beam. Nonlinear Dynamics 82: 1721-1742

[33] Sahoo B, Panda L N and Pohit G 2016 Combination, principal parametric and internal resonances of an accelerating beam under two frequency parametric excitation. International Journal of Non-Linear Mechanics 78: 35-44

[34] Mao X Y, Ding H and Chen L Q 2017 Dynamics of a supercritically axially moving beam with parametric and forced resonance. Nonlinear Dynamics 89: 1475-1487

[35] Zhang D B, Tang Y Q and Chen L Q 2017 Irregular instability boundaries of axially accelerating viscoelastic beams with 1:3 internal resonance. International Journal of Mechanical Sciences 133: 535-543

[36] Wang B 2018 Effect of rotary inertia on stability of axially accelerating viscoelastic Rayleigh beams. Applied Mathematics and Mechanics 39: 717-732

[37] Lv H W, Li L and Li Y H 2018 Non-linearly parametric resonances of an axially moving viscoelastic sandwich beam with time-dependent velocity. Applied Mathematical Modelling 53: 83-105

[38] Brandenburg G 1973 A mathematical model of a continuous elastic web in a system of driven, looped, rollers. Control Engineering and Data Processing 3: 62-69 